\title{
Reversible supraventricular tachycardia complicating diabetic ketoacidosis in an adult patient: A case report
}

\author{
Uchenna Okechukwu Ugwuneji ${ }^{1}$, Ifeanyi Ucha Julius ${ }^{2}$, Yakubu Lawal ${ }^{2}$, Rifkatu Reng Sonnie ${ }^{2}$, \\ Ochai Attai Ateko ${ }^{1}$, Felicia Ehusani Anumah ${ }^{3}$ \\ From ${ }^{I}$ Senior Registrar, ${ }^{2}$ Consultant Endocrinologist, ${ }^{3}$ Professor of Endocrinology, Department of Internal Medicine, College of Health Sciences, \\ University of Abuja, Nigeria
}

\begin{abstract}
Diabetic ketoacidosis (DKA) is a commonly encountered serious acute metabolic complication of diabetes mellitus (DM) in adolescents and young adults. It is traditionally associated with poorly treated or newly diagnosed type $1 \mathrm{DM}$, however, in the setting of type 2 $\mathrm{DM}$, inadequate insulin treatment, non-compliance to treatment, newly diagnosed DM, acute illnesses, drugs, and extreme stress can precipitate DKA. We report the case of a 42-year-old known diabetic of 7 years duration with a family history of DM who presented with a two-week history of difficulty in breathing, polyuria, and vomiting. On further examination, pulse rate was 220 beats per minute, respiratory rate 40 cycles per minute, temperature $38.4^{\prime} \mathrm{C}$. Random blood sugar was $18.1 \mathrm{mmol} / \mathrm{l}$ with ketonuria ++ . Severe acidosis and mild hypokalemia were noted with her electrocardiogram (ECG) showing supraventricular tachycardia. She was managed and discharged in stable condition with a normal ECG after 20 days on admission to continue basal and pre-meal insulin at home. Adequate diabetic education was conducted and follow-up with endocrinology and cardiology units was advised.
\end{abstract}

Key words: Electrocardiogram, Diabetic ketoacidosis, Supraventricular tachycardia, Beta-blockers

$\mathrm{D}$ iabetic ketoacidosis (DKA) is an acute life-threatening emergency of diabetes mellitus (DM) typically characterized by acidosis, ketosis, and usually hyperglycemia. It can be precipitated by acute illnesses (sepsis, acute myocardial infarction, and stroke), medications (sodiumglucose co-transporter 2 inhibitors), and non-adherence to therapy, or insulin [1-4]. The complications of DKA results from mainly severe dehydration, metabolic acidosis, and electrolyte derangement. Although electrolyte abnormalities are common in DKA, cardiac arrhythmias, however, are rare and are usually due to metabolic acidosis and dyselectrolytaemias such as hypokalemia and hypophosphatemia [5]. The effects of acidosis on the heart are variable and $\mathrm{pH}$-dependent. Severe acidosis causes a depressing effect on the heart and may be associated with decreased contractility and a predisposition to cardiac arrhythmias. On the other hand, mild-to-moderate acidosis can lead to increased cardiac contractility and peripheral vascular resistance also predisposing to disturbances in cardiac rhythm. Disturbances in repolarization including early or delayed repolarization, atrioventricular nodal delay, and re-entry are

\section{Access this article online}

Received - 14 August 2021

Initial Review - 31 August 2021

Accepted - 18 September 2021

DOI: $10.32677 /$ ijcr.v7i10.3044 commonly encountered cardiac electrical disturbances triggered by acidosis [6-10]. We report the case of a 42-year-old teacher; known diabetic for 7 years managed by the Endocrinology unit but was lost to follow-up for 4 years. The relevance of this case report is to raise awareness of physicians to the occurrence of arrhythmias as a complication of acidosis or electrolyte imbalances which are characteristic of DKA. Thus, highlighting the importance of performing an electrocardiogram in all patients who present with a hyperglycemic emergency. Detection of abnormal rhythms such as supraventricular tachycardia (SVT) and treating them appropriately might improve outcomes in DKA.

\section{CASE REPORT}

A 42-year-old female was brought into the medical emergency room with complaints of worsening difficulty in breathing on exertion and fever of two weeks duration. She also presented with vomiting for 2 days, about 10 episodes daily, and approximately $50 \mathrm{mls}$ per episode composed of ingested food materials, nonbloody, and non-bilious. There was also associated increased urinary frequency with a day/night ratio of $4 / 5$ as opposed to a previous $3 / 12$ months previously. The estimated daily urine output was $3500 \mathrm{ml}$ per day and there was an associate frothiness

Correspondence to: Dr. Yakubu Lawal, Department of Internal Medicine, College of Health Sciences, University of Abuja, Nigeria. E-mail: lawalyaqub2006@yahoo.com

(C) 2021 Creative Commons Attribution-NonCommercial 4.0 International License (CC BY-NC-ND 4.0). 
of urine of a month duration. There were also features of peripheral neuropathy namely recurrent numbness and tingling sensation in the feet for about two years prior to presentation. The patient was a known diabetic for 7 years. There was also associated family history of diabetes in her mother and brother as well as hypertension in her mother.

At presentation, she was conscious but lethargic with a Glasgow Coma Score of 13/15 (EO-4, BVR-5, and BMR-4). She was febrile with a temperature of $38.4^{\circ} \mathrm{C}$, tachypneic ( 40 cycles per minute) with associated acidotic breathing, pulse rate was 220 beats per minute, and blood pressure was $124 / 78 \mathrm{mmHg}$.

Random blood sugar at presentation was $18.1 \mathrm{mmol} / \mathrm{L}$ with HbAlc 9.3\%. The urine dipstick revealed ketones +++ , glucose ++ , and blood ++ while effective osmolality was $306 \mathrm{mosm} /$ $\mathrm{kg}$ with an anion gap of $20.2 \mathrm{mmol} / \mathrm{L}$. Electrolytes, urea, and creatinine $(\mathrm{E} / \mathrm{U} / \mathrm{Cr})$ on admission and following recovery are shown in Table 1. Blood culture, urine, sputum, stool microscopy, and culture, showed no bacterial growth; cardiac troponin was I: 0.16 (normal); and fasting lipid profile was within normal limits. Electrocardiogram (ECG) showed SVT (Fig. 1). A clinical assessment of diabetic ketoacidosis (DKA) precipitated by sepsis (chest infection) with supraventricular tachycardia was made.

Treatment was commenced with crystalloids (normal saline) and intramuscular hourly insulin, later converted to glucose, insulin, and potassium chloride infusion with hourly blood glucose monitoring. Intravenous antibiotic (meropenem) was commenced with metronidazole. Intensive care was advised for the patient but was declined for personal reasons. Digoxin $(0.25 \mathrm{mg})$ daily and bisoprolol $(10 \mathrm{mg})$ daily were commenced.

Four days after admission, his electrolytes were normalized, blood sugar was controlled, and SVT was reversed (Fig. 2). She was discharged a week later on insulin glargine and metformin, to visit the endocrinology and cardiology clinic. Adequate and comprehensive diabetic medical education was carried out, including self-monitoring of blood glucose, medical nutritional therapy, hypoglycemia management, and foot care.

\section{DISCUSSION}

In DKA, the types of cardiac arrhythmias seen are variable and include re-entry, atrial fibrillation, ventricular and supraventricular tachycardia, premature atrial, and ventricular complexes. The occurrence of SVT in DKA is rare as there are a few case reports published by Faruqi et al. in 2015 [11], Finn et al. [12] and AyonAguilar et al. in 2018 [13], and Thomas et al. in 2007 [14].

SVT occurring in the background of DKA is uncommon. All the cases identified so far, except for the case report by Finn et al. [12], occurred in females. Concerning the age of occurrence, most reported cases were between ages 12 and 14 years and only one incidence at age 29 years was described by Ayon-Aguilar et al. [13]. This contrasts our index case who was a 42-years-old, representing the oldest person described in the literature searched. The index case had a family history of DM, in addition to the treatment history, consistent with type $2 \mathrm{DM}$ which is similar to the case reported by Ayon-Aguilar et al. [13] However, the majority of other cases reported in the literature had type $1 \mathrm{DM}$.

The index case had both severe acidosis and mild hypokalaemia. This can predispose to SVT via early or delayed repolarization, atrioventricular nodal delay, or re-entry [6,7]. This is in keeping with some of the rare reports of DKA causing cardiac dysrhythmias [11-14]. Four out of these five cases from previous reports presented initially with bicarbonate of $<10 \mathrm{meq} / 1$, while three out of the five had hypokalemia and $\mathrm{pH}$ of $<7.2$ with only Finn et al. [12] reporting hyperkalemia (5.2 mmol/1) [11-14].

Concerning treatment, cardioversion was most commonly achieved with intravenous adenosine, however, Faruqi et al. [11] reported a case that responded to vagal maneuvers, while AyonAguilar et al. [13] documented a case that received flecainide to restore sinus rhythm. This is in contrast to the index case where bisoprolol and digoxin were used. In all cases, management of the hyperglycemic emergency with electrolyte correction and synchronized cardioversion caused resolution of SVT.

These reports of SVT and other arrhythmias in DKA leave some unanswered questions. Despite the widespread electrolyte abnormalities that are known causes of arrhythmias, why do only a few individuals encounter this? Are there particular predisposing factors to the development of these electrical disturbances such as early cardiac autonomic neuropathy? The answer to these questions lies in more research into this area.

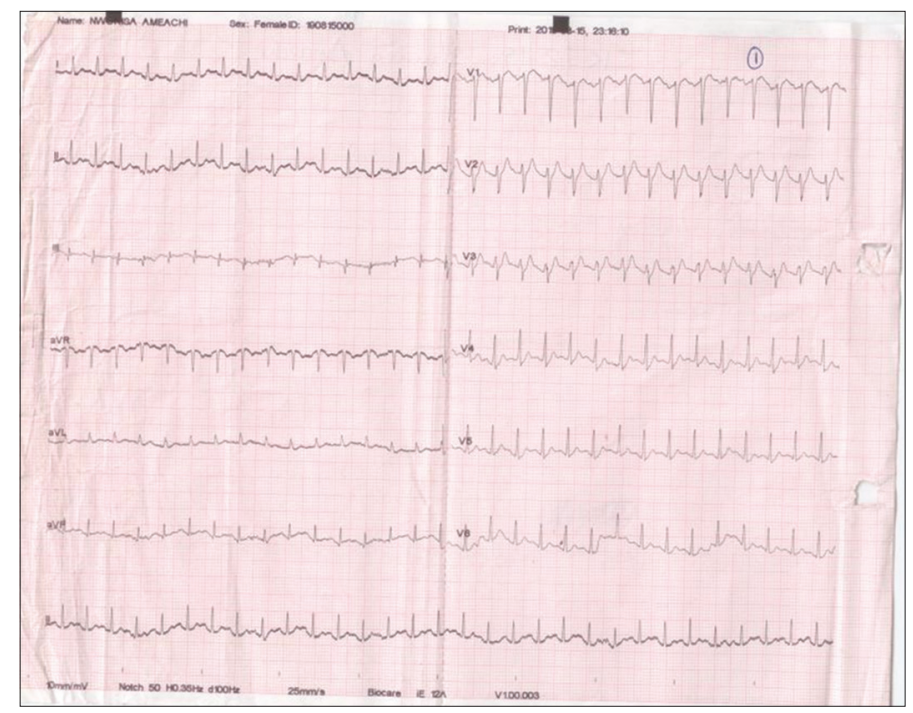

Figure 1: The ECG of the patient on admission showing supraventricular tachycardia and T-wave abnormality

Table 1: Electrolytes, urea, and creatinine of the patient on admission and following recovery

\begin{tabular}{|c|c|c|c|c|c|c|c|}
\hline Normal ranges & $\begin{array}{c}\mathrm{Na}(136-148) \\
\mathrm{mmol} / \mathrm{L}\end{array}$ & $\begin{array}{c}\mathrm{K}(3.5-5.0) \\
\mathrm{mmol} / \mathrm{L}\end{array}$ & $\begin{array}{c}\mathrm{Cl}(98-110) \\
\mathrm{mmol} / \mathrm{L}\end{array}$ & $\begin{array}{c}\mathrm{HCO}_{3}(20- \\
30) \mathrm{mmol} / \mathrm{L}\end{array}$ & $\begin{array}{c}\text { Urea (2.1- } \\
7.1) \mathrm{mmol} / \mathrm{L}\end{array}$ & $\begin{array}{c}\text { Creatinine } \\
(49-90) \text { umol/L }\end{array}$ & $\begin{array}{c}\operatorname{eGFR}(\mathrm{ml} / \\
\left.\mathrm{min} / 1.73 \mathrm{~m}^{2}\right)\end{array}$ \\
\hline On admission & 149 & 3.4 & 114 & $<10$ & 9.0 & 66 & 114.32 \\
\hline 4 days post-admission & 142 & 4.2 & 103 & 23 & 6.5 & 44 & 138.26 \\
\hline
\end{tabular}




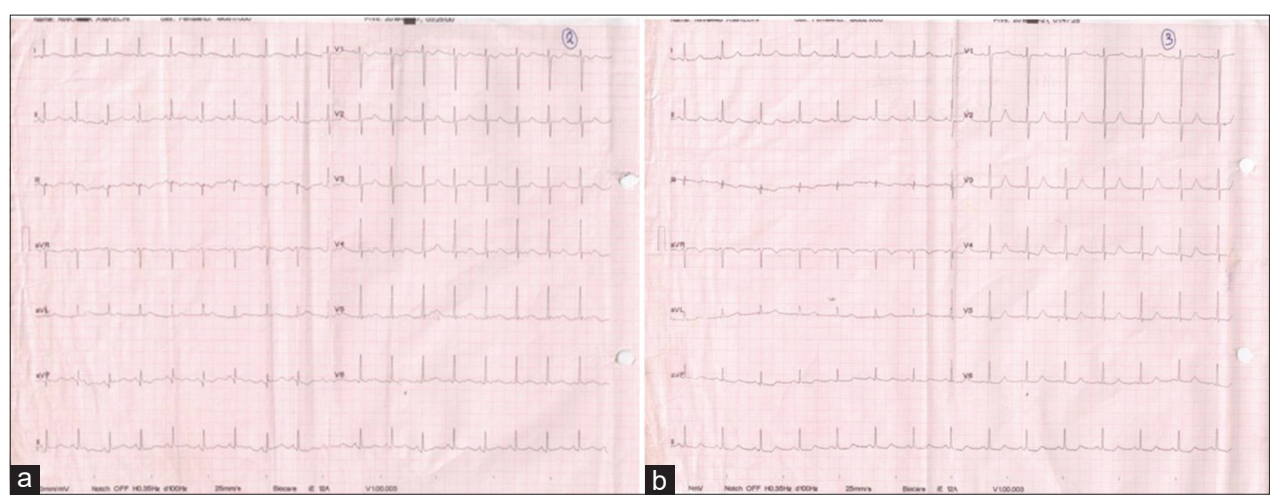

Figure 2: (a) The ECG of the patient during recovery with sinus tachycardia, short PR interval, T-wave abnormality; (b) The patient's ECG showing sinus rhythm after recovery

\section{CONCLUSION}

Clinicians should be on high alert to recognize any form of arrhythmia including SVT in persons presenting with DKA. Electrocardiography should be routinely performed so as to recognize any form of arrhythmia early and appropriately intervene. Diligent resuscitation of persons with DKA can assist in early recovery from SVT.

\section{REFERENCES}

1. Zayed H. Epidemiology of diabetic ketoacidosis in Arab patients with Type 1 diabetes: A systematic review. Int J Clin Pract 2016;70:186-95.

2. Usher-Smith JA, Thompson M, Ercole A, Walter FM. Variation between countries in the frequency of diabetic ketoacidosis at first presentation of Type 1 diabetes in children: A systematic review. Diabetologia 2012;55:2878-94.

3. Jensen ML, Persson F, Andersen GS, Ridderstråle M, Nolan JJ, Carstensen B, et al. Incidence of ketoacidosis in the danish type 2 diabetes population before and after introduction of sodium-glucose cotransporter 2 inhibitorsda nationwide, retrospective cohort study, 1995-2014. Diabetes Care 2017;40:e57-8.

4. Westphal SA. The occurrence of diabetic ketoacidosis in non-insulindependent diabetes and newly diagnosed diabetic adults. Am J Med 1996;101:19-24.

5. Cubbon RM, Kearney MT. Review: Acute metabolic derangement and the heart. Br J Diabetes Vasc Dis 2007;7:218-22.

6. Nisbet AM, Burton FL, Walker NL, Craig MA, Cheng H, Hancox JC, et al. Acidosis slows electrical conduction through the atrio-ventricular node. Front Physiol 2014;5:1-8.
7. Youssef OI, Farid SM. QTc and QTd in children with Type 1 diabetes mellitus during diabetic ketoacidosis. ISRN Pediatr 2012;2012:1-4.

8. Handy JM, Soni N. Physiological effects of hyperchloraemia and acidosis. Br J Anaesth 2008;101:141-50.

9. Schwartz A, Brotfain E, Koyfman L, Kutz R, Gruenbaum SE, Klein M, et al. Association between hypophosphatemia and cardiac arrhythmias in the early stage of sepsis : Could phosphorus replacement treatment reduce the incidence of arrhythmias ? Electrolyte Blood Press 2014;5997:19-25.

10. Miszczuk K, Mroczek-wacinska J, Piekarski R, Lukasik BW, Jawniak R. Ventricular bigeminy and trigeminy caused by hypophosphataemia during diabetic ketoacidosis treatment : A case report. Ital J Pediatr 2019;45:42.

11. Faruqi TA, Hanhan UA, Orlowski JP, Laun KS, Williams AL, Fiallos MR. Supraventricular tachycardia with underlying atrial flutter in a diabetic ketoacidosis patient. Clin Diabetes 2015;33:146-9.

12. Finn BP, Fraser B, O’Connell SM. Supraventricular tachycardia as a complication of severe diabetic ketoacidosis in an adolescent with newonset Type 1 diabetes. BMJ Case Rep 2018;2018:222861.

13. Ayón-Aguilar J, Valladares-Villalobos J, Santos-López G, Martínez S. Diabetic ketoacidosis complicated by supraventricular tachycardia in a young adult: A case report. Invest Clin 2018;59:155-60.

14. Thomas N, Scanlon J, Ahmed M. Supraventricular tachycardia in association with diabetic ketoacidosis. Br J Diabetes Vasc Dis 2007;7:244-5.

Funding: None; Conflicts of Interest: None Stated.

How to cite this article: Ugwuneji UO, Julius IU, Lawal Y, Sonnie RR, Ateko OA, Anumah FE. Reversible supraventricular tachycardia complicating diabetic ketoacidosis in an adult patient: A case report. Indian J Case Reports. 2021;7(10):427-429. 